\title{
Factors Associated with Depression and Sub-Dimension Symptoms in Adolescent Narcolepsy
}

\author{
Yang Yang ${ }^{1, *}$ \\ Chenyang $\mathrm{Li}^{2} * *$ \\ Long Zhao ${ }^{2}$ \\ Jing $\mathrm{Li}^{2}$ \\ Fang $\mathrm{Han}^{2}$ \\ Fulong $\mathrm{Xiao}^{3}$
}

'Department of Neuropsychiatry and Behavioral Neurology and Clinical Psychology, Beijing Tiantan Hospital, Capital Medical University, Beijing, 100050, People's Republic of China; ${ }^{2}$ Sleep Medicine Center, Department of Respiratory and Critical Care Medicine, Peking University People's Hospital, Beijing, 100044, People's Republic of China; ${ }^{3}$ Department of General Internal Medicine, Peking University People's Hospital, Beijing, I00044, People's Republic of China

*These authors contributed equally to this work
Correspondence: Fang Han; Fulong Xiao Peking University People's Hospital, No. II, South Avenue, Beijing, People's Republic of China

Email hanfangl@hotmail.com; xiaofulong168I@163.com
Objective: We evaluate the association between depression symptoms, clinical features (disease onset-age, disease duration, sleep-related hallucination), sleepiness, and polysomnography parameters in adolescent narcolepsy type 1 patients.

Methods: Eighty-three adolescent narcolepsy type 1 patients were involved in this crosssectional study. Patients completed questionnaires evaluating depression symptoms (Center for Epidemiologic Studies Depression Scale) and sleepiness (Epworth Sleepiness Scale). Parameters from polysomnography and multiple sleep latency test were also collected.

Results: Patients with depression symptoms (62.7\%) have later disease onset-age. Depression symptoms were associated with sleep-related hallucination $(\mathrm{OR}=2.75)$. Six independent variables were associated with sub-dimensional depression symptoms, including sleep latency, sleep efficiency, sleep-related hallucination, Epworth sleepiness scale, disease duration, and disease onset-age.

Conclusion: Sleep-related hallucination is associated with total depression symptoms in adolescent narcolepsy. Subjective sleepiness is associated with depressed affect, somatic symptoms, and interpersonal problems. Lower sleep efficiency is associated with a lack of positive affect.

Keywords: narcolepsy, adolescents, depression symptoms, hypnagogic/hypnopompic hallucination

\section{Introduction}

Narcolepsy type 1 is a central sleepiness disorder, which is characterized by excessive daytime sleepiness, cataplexy, hypnagogic/hypnopompic hallucination (or sleep-related hallucination), and sleep paralysis. Hypocretin deficiency in the hypothalamus due to autoimmune response is thought to be the pathophysiology of narcolepsy type 1. Depression is a common but serious mental disorder in narcolepsy. ${ }^{1}$ A follow-up study found that depression symptoms can interfere with health-related quality of life in narcolepsy patients. ${ }^{2}$ The high presence of depression symptoms/moods in narcolepsy has been reported in many studies, but the exact incidence of depression or depression symptoms in narcolepsy is not clear and still controversial. ${ }^{3-7}$ Moreover, due to the overlap between depression symptoms and narcolepsy, it is difficult to separate depression symptoms into independent clinical entities. Several studies have suggested that narcolepsy and depression can interact and influence each other. ${ }^{8,9}$ It is supposed that some narcolepsy patients are recognized as depression symptoms before narcolepsy, while depression 
symptoms may come along with narcolepsy diagnosis. Medical treatment also has mood-modifying effects. ${ }^{10}$ A cross-sectional study showed that narcolepsy patients treated with stimulants and anti-cataplectic drugs reported more depression symptoms and poor quality of life than those treated with stimulants alone. ${ }^{11}$

Depression symptoms typically begin during adolescence, which is an important stage in growth and development. If left ignored, depression symptoms may have serious consequences such as poor school performance, ${ }^{12}$ obesity, ${ }^{13,14}$ and suicide. ${ }^{15,16}$ Even more significant is that sub-dimensions in depression symptoms, such as lack of positive affect and interpersonal problems, may be one of the most prominent manifestations in adolescent depression. Therefore, evaluating factors associated with depression symptoms in adolescent narcolepsy is significant for clinical practice to optimize patients' outcomes. Factors that may be associated with depression symptoms include onset-age, sleepiness severity, disease duration, and obesity. ${ }^{17}$ In this study, the impact and significance of factors on depression and sub-dimensional symptoms were evaluated in adolescent narcolepsy type 1 patients.

\section{Methods}

\section{Participants}

Eighty-three drug-naïve adolescent narcolepsy type 1 patients were recruited from the Sleep Medicine Center at Peking University People's Hospital. All narcolepsy type 1 patients were diagnosed according to the International Classification of Sleep Disorders -3 (ICSD$3):{ }^{18}$ the presence of daytime sleepiness lasting at least 3 months, typical cataplexy, with mean sleep latency $\leq 8$ minutes and 2 or more sleep-onset REM periods (SOREMPs) on the Multiple Sleep Latency Test (MSLT). Or lower levels of orexin $(<110 \mathrm{pg} / \mathrm{mL})$ in cerebral spinal fluid. All the patients had cataplexy and carried HLADQB1*06:02 gene. All participants or their parents or legal guardians were provided written informed consent, and this study was conducted in accordance with the Declaration of Helsinki. This study was approved by the ethics committees of Peking University People's Hospital.

\section{Polysomnography}

All narcolepsy patients underwent one overnight polysomnography (PSG, Respironics LE-Series Physiological Monitoring System, Alice 6 LE, FL, USA) with recording starting from $10 \mathrm{pm}$ to $6 \mathrm{am}$ the next morning. Following the guideline of the American Academy of Sleep Medicine (AASM), standard electroencephalogram derivations, chin electromyogram, electro-oculogram, and electrocardiogram were recorded. Oral and nasal airflow, snoring, chest and abdominal breathing, oxygen saturation, and body position were also recorded. The PSG recordings were visually scored according to the AASM manual for Scoring Sleep and Associated Events ${ }^{19}$ by assigning one of five stages (N1, N2, N3, REM, or Wake) to every 30-s epoch of the data. Obstructive apnea was defined as a reduction in airflow $\geq 90 \%$ lasting at least $10 \mathrm{~s}$ and associated with persistent respiratory effort; hypopnea was defined as a reduction in airflow $\geq 30 \%$ lasting at least $10 \mathrm{~s}$ and accompanied by a $4 \%$ or greater oxygen desaturation. The apnea-hypopnea index (AHI) was calculated as the average of the total number of apnea and hypopnea events experienced per hour of sleep.

Multiple sleep latency test (MSLT) was performed on the next day after the overnight PSG. A total of five naps were scheduled at 2-h intervals. Sleep latency was defined as the time that elapsed from the start of the test to the first 30 -s epoch scored as sleep. Each MSLT was ended $20 \mathrm{~min}$ after the onset of sleep or after 20 min of wakefulness. The average sleep latency from the five naps was calculated.

Periodic leg movement in sleep (PLMS) was defined and scored using standard criteria from the AASM manual for Scoring Sleep and Associated Events: ${ }^{19}$ a series of four consecutive leg movements lasting $0.5-5 \mathrm{~s}$ with an amplitude $\geq 8 \mu \mathrm{v}$ above the resting baseline and separated by intervals of 5-90 s. Leg movement was not scored if it occurred during a period from $0.5 \mathrm{~s}$ preceding apnea, hypopnea, or respiratory event-related arousal to 0.5 $\mathrm{s}$ following such an event. The PLMS index (PLMSI) was quantified as the frequency of PLMS per hour of sleep. In this study, patients were divided into two groups based on the presence or absence of PLMS according to the ICSD -3 in adolescents: ${ }^{18}$ with PLMS (PLMI > 5) and without PLMS (PLMI $\leq 5$ ).

\section{Questionnaires and Clinical Data}

Information about age, gender, body mass index (BMI), age at narcoleptic symptom onset (or disease onset-age), disease duration, hypnagogic/hypnopompic hallucinations, and Epworth Sleepiness Score (ESS) were collected from narcolepsy patients. The Center for Epidemiologic Studies Depression Scale (CES-D) of Chinese version was used to measure depression symptoms in adolescent narcolepsy patients. ${ }^{20}$ CES-D is a 20-item scale and there are 
4-point response options ( $0-3$ points) for each item. Total scores range from 0 to 60 and higher scores indicate increasing levels of depression symptoms, with a cut-off score $\geq 20$ suggesting depression disorder or depression feelings/symptoms. ${ }^{21}$ Moreover, a total of four subdimensional depression symptoms, including depressed affect, lack of positive affect, somatic symptoms, and interpersonal problems, can be identified in the CES-D score. ${ }^{20}$ Therefore, two kinds of depression symptoms measurements were used in our study: one is the cut-off point for depression symptoms (CES-D score $\geq 20$ ) and the other is four sub-dimensional depression symptoms.

\section{Statistical Analysis}

The convergence and dispersion trends for quantitative variables were expressed as mean \pm SD deviation and qualitative variables were expressed as frequencies and percentages. First, the demographic variables and PSG parameters were compared between patients with and without depression symptoms. Continuous variables were compared using the two independent-samples $t$-test. Categorical variables were compared using $\chi^{2}$ test. Spearman correlation coefficients were then performed to investigate the correlation between the four sub-dimensional CES-D scores, demographic variables, and PSG parameters.

To determine the associated factors in adolescent narcolepsy patients' total depression symptoms, multiple logistic regression with backwards stepwise selection was conducted first, where the total CES-D score was transformed as a binary variable (according to the cut-off point $\geq 20$ ). Then, four separate multiple linear regressions using stepwise backward selection were performed to evaluate the association between the four sub-dimensional CES-D scores with demographic variables and PSG parameters. Statistical tests were two-tailed and the significance level was set at $P<$ 0.05. The Durbin-Watson test was used to examine the normality assumption for each model. All statistical analyses were conducted using SPSS (Version 23).

\section{Results}

\section{Subjects Characteristics}

For 83 narcolepsy patients in this study, $62.7 \%$ of them $(\mathrm{n}=$ 52) reported depression symptoms (CES-D score $\geq 20$ ) and $45.8 \%$ of narcolepsy patients $(n=38)$ reported hypnagogic/ hypnopompic hallucination and $30 \%$ of narcolepsy patients $(\mathrm{n}=30)$ had sleep paralysis (Table 1). Hallucinations in narcolepsy are sleep-related, which occur at the onset of sleep, are defined as hypnagogic hallucinations, and those that occur during awakening are referred to as hypnopompic hallucinations. A typical hallucination in narcolepsy is the sense that a threatening stranger is in the bedroom or that one is being attacked by animals. $27.7 \%$ of narcolepsy patients $(n=23)$ had PLMS according to the ICSD-3 criteria (Table 1). Although patients with depression symptoms seemed to have higher hypnagogic/hypnopompic hallucination percentage and more severity subjective sleepiness (namely, higher ESS) than those without depression symptoms, these differences were not statistically significant (Table 1). All patients showed three peaks of disease onsetage, with one peak around 6-8 years, the second peak around 10 years, and the other peak around $12-13$ years (Figure 1A). However, patients with depression symptoms had more subjects with onset-age around 6-8 years and a much later onset-age around 10-15 years than those without depression symptoms (Figure 1B).

\section{Determinants of Total Depression Symptoms in Narcolepsy Patients}

As a result of group differences between patients with depression symptoms and patients without depression symptoms, variables achieving significance at a level of $P<0.15$ were entered into the logistical regression. A total of six independent variables were adjusted in the logistical regression model, including age, disease duration, education level, hypnagogic/hypnopompic hallucination, apneahypopnea index, and ESS. Multivariate analysis showed that only hypnagogic/hypnopompic symptoms were associated with total depression symptoms in adolescent narcolepsy patients (Table 1).

\section{Determinants of Sub-Dimension Depression Symptoms in Narcolepsy Patients}

Results from four separate multiple linear regression models with sub-dimensional CES-D scores (depressed affect, lack of positive affect, somatic symptoms, and interpersonal problems) as the dependent variables are shown in Table 2. Variables that are correlated with subdimensional CES-D scores at a significant level of $P<$ 0.2 (Supplementary Table 1) and the binary variables with or without hypnagogic/hypnopompic hallucination were included in the linear regression model.

A total of seven independent variables were adjusted in the multiple linear regression model for depressed affect 
Table I Demographic, Clinical Characteristics, and Polysomnography Parameters Associated with Depression Symptoms in Adolescent Narcolepsy Patients

\begin{tabular}{|c|c|c|c|c|c|}
\hline & \multicolumn{3}{|c|}{ Univariate } & \multicolumn{2}{|c|}{ Multivariate } \\
\hline & $\begin{array}{l}\text { Without Depression Symptoms } \\
\qquad(n=3 I)\end{array}$ & $\begin{array}{l}\text { With Depression Symptoms } \\
\qquad(\mathrm{n}=52)\end{array}$ & $P$ value & B & OR $(95 \% \mathrm{Cl})$ \\
\hline Male/Female & $22 / 9$ & $29 / 23$ & 0.169 & - & - \\
\hline Age & $13(8,15)$ & $14(11,15)$ & 0.141 & - & - \\
\hline Disease onset-age & $7(5.6,10)$ & $8(6,11.8)$ & 0.317 & - & - \\
\hline Disease duration (years) & I $(0.4,9)$ & $4(1.3,7)$ & 0.106 & - & - \\
\hline BMI & $22.6 \pm 5.4$ & $23.9 \pm 4.9$ & 0.237 & - & - \\
\hline Education level (years) & $7(2,9)$ & $8(5,9)$ & 0.147 & - & - \\
\hline With hallucination & 10 & 28 & 0.056 & 1.01 & $2.75(1.1,7.3)$ \\
\hline With sleep paralysis & 7 & 18 & 0.248 & & \\
\hline Sleep efficiency & $88.2 \pm 10.4$ & $86.5 \pm 12.2$ & 0.529 & - & - \\
\hline $\mathrm{NI}+\mathrm{N} 2 \%$ & $55(49.4,62)$ & $53.6(45.5,63.3)$ & 0.843 & - & - \\
\hline $\mathrm{R} \%$ & $22.4(18.1,29.7)$ & $22.2(16.5,27.8)$ & 0.77 & - & - \\
\hline N3\% & $22.3(16.6,25.8)$ & $21(14.9,26.3)$ & 0.873 & - & \\
\hline Arousal index & II.6 (8.9, I7.8) & II.2 (7.3, 16.2) & 0.44 & - & - \\
\hline With PLMS & 12 & 11 & 0.2 & - & - \\
\hline Sleep latency & $2.8(1.8,3.5)$ & $1.9(0.9,4.1)$ & 0.285 & - & - \\
\hline $\mathrm{AHI}$ & $1.3(0.3,3.2)$ & $2.1(1.1,3.4)$ & 0.087 & - & - \\
\hline ESS & $16.1 \pm 4.5$ & $17.6 \pm 3.7$ & 0.093 & - & - \\
\hline
\end{tabular}

Notes: Multivariate logistic regression model, adjusted for age, disease duration, education level, hypnagogic/hypnopompic hallucination, apnea-hypopnea index, and ESS. Abbreviations: BMI, body mass index; AHI, apnea-hypopnea index; ESS, Epworth sleepiness scale; PLMS, periodic limb movement in sleep; Cl, confidence interval.

scores, including hypnagogic/hypnopompic hallucination, ESS, disease onset-age, BMI, education level, sleep latency, and disease duration. Five independent variables were adjusted in the multiple linear regression model for lack of positive affect scores, including hypnagogic/hypnopompic hallucination, sleep efficiency, disease duration, arousal index, and BMI. Four independent variables were adjusted in the multiple linear regression model for somatic symptom scores, including hypnagogic/hypnopompic hallucination, education level, ESS, and sleep latency. Seven independent variables were adjusted in the multiple linear regression model for interpersonal problem scores, including hypnagogic/hypnopompic hallucination, education level, ESS, disease duration, sleep efficiency, AHI, and BMI.

Patients with hypnagogic/hypnopompic hallucination showed significantly more severity in somatic symptoms than those without hypnagogic/hypnopompic hallucination
(Figure 2). More severe subjective sleepiness (namely, higher ESS) is associated with depressed affect, somatic symptoms, and interpersonal problems (Table 2). Additionally, longer disease duration is associated with higher depressed affect score and interpersonal problems (Table 2). Moreover, patients with later disease onset-age and shortened sleep latency seem to have higher depressed affect score (Table 2). Lower sleep efficiency is associated with a lack of positive affect (Table 2).

\section{Discussion}

This study evaluated factors associated with depression symptoms in adolescent narcolepsy. We found that patients with hypnagogic/hypnopompic hallucination were more frequent in total depression symptoms. ESS, disease duration, disease onset-age, sleep latency, and sleep efficiency were associated with sub-dimensional depression symptoms. 
A

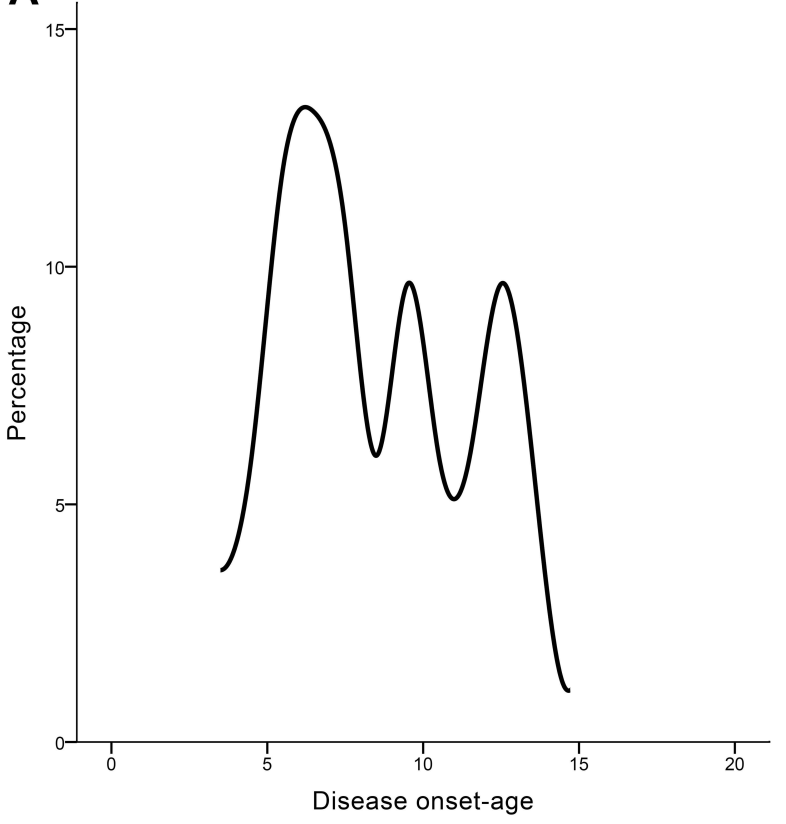

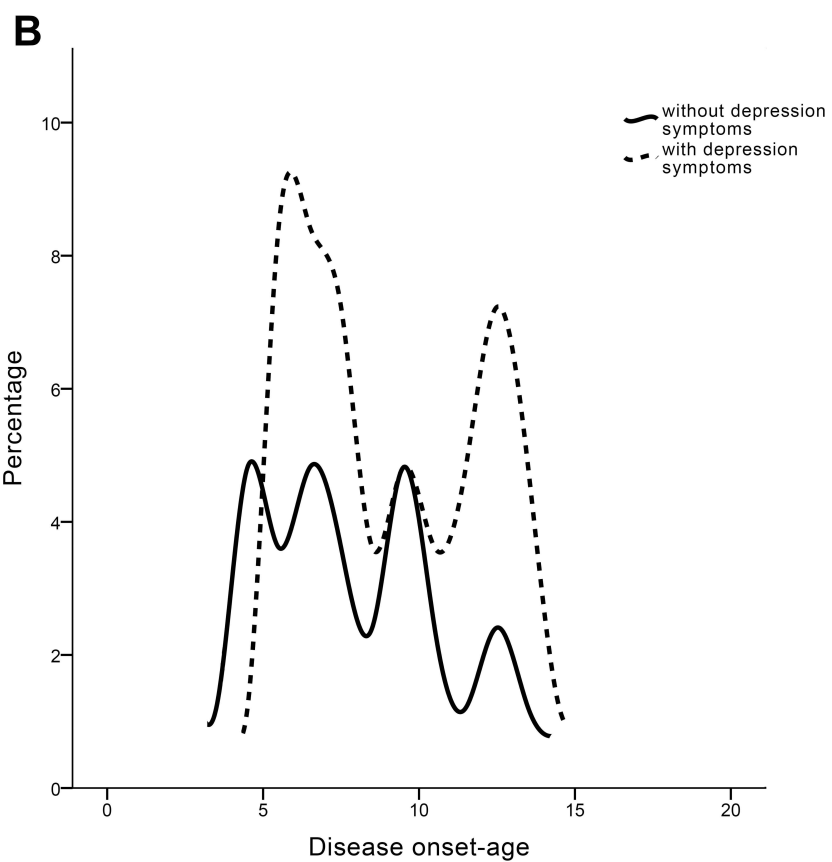

Figure I The distribution of disease onset-age in all adolescent narcolepsy patients (A) and patients with/without depression symptoms respectively (B).

The association between total depression symptoms and sleep-related hallucination in this study is similar to findings in previous adult narcolepsy studies. ${ }^{11,22}$ There are few reports of factors associated with adolescent narcolepsy depression symptoms, and especially for sleeprelated hallucination. Further analysis demonstrated that patients with sleep-related hallucination exhibit much higher score in depressed affect, lack of positive affect and somatic symptoms. One published study revealed depression's relationship to hypnagogic/hypnopompic hallucination can be partially explained by anxiety in adults, ${ }^{23}$ but it is difficult to measure the degree of anxiety in adolescents, especially for those with hypnagogic/hypnopompic hallucination. Therefore, further studies should

Table 2 Factors Associated with Sub-Dimension Depression Symptoms in Adolescent Narcolepsy Patients

\begin{tabular}{|c|c|c|c|c|c|c|}
\hline & Sleep Latency & Sleep Efficiency & Hallucination & ESS & $\begin{array}{c}\text { Disease } \\
\text { Duration }\end{array}$ & $\begin{array}{c}\text { Disease Onset- } \\
\text { Age }\end{array}$ \\
\hline & $\begin{array}{c}\text { Standard } \\
\text { Coefficient } 95 \% \\
\text { Cl }\end{array}$ & $\begin{array}{c}\text { Standard } \\
\text { Coefficient } 95 \% \\
\text { Cl }\end{array}$ & $\begin{array}{c}\text { Standard } \\
\text { Coefficient } 95 \% \\
\text { Cl }\end{array}$ & $\begin{array}{c}\text { Standard } \\
\text { Coefficient } 95 \% \\
\mathrm{Cl}\end{array}$ & $\begin{array}{c}\text { Standard } \\
\text { Coefficient } 95 \% \\
\mathrm{Cl}\end{array}$ & $\begin{array}{c}\text { Standard } \\
\text { Coefficient } 95 \% \\
\text { Cl }\end{array}$ \\
\hline $\begin{array}{l}\text { Depressed } \\
\text { affect }\end{array}$ & $\begin{array}{c}-0.212(-1.223 \\
-0.017)\end{array}$ & - & $\begin{array}{c}0.244(0.45 I \\
5.123)\end{array}$ & $\begin{array}{c}0.259(0.083 \\
0.646)\end{array}$ & $0.24(0.017,0.729)$ & $\begin{array}{c}0.308(0.126 \\
0.872)\end{array}$ \\
\hline $\begin{array}{l}\text { Lack of } \\
\text { positive affect }\end{array}$ & - & $\begin{array}{c}-0.242(-0.115 \\
-0.008)\end{array}$ & $0.22(0.047,2.514)$ & - & - & - \\
\hline $\begin{array}{l}\text { Somatic } \\
\text { symptoms }\end{array}$ & - & - & $0.305(0.803,4.11)$ & $\begin{array}{c}0.266(0.064 \\
0.463)\end{array}$ & - & - \\
\hline $\begin{array}{l}\text { Interpersonal } \\
\text { problems }\end{array}$ & - & - & - & $0.309(0.05,0.228)$ & $\begin{array}{c}0.298 \\
(0.049,0.247)\end{array}$ & - \\
\hline
\end{tabular}

Notes: Model for depressed effect, adjusted for hypnagogic/hypnopompic hallucination, ESS, disease onset-age, BMI, education level, sleep latency, and disease duration. Model for lack of positive affect, adjusted for disease duration, BMI, sleep efficiency, arousal index, and hallucination. Model for somatic symptoms, adjusted for hypnagogic/ hypnopompic hallucination, education level, ESS, and sleep latency. Model for interpersonal problems, adjusted for hypnagogic/hypnopompic hallucination, education level, ESS, disease duration, sleep efficiency, AHI, and BMI.

Abbreviations: ESS, Epworth sleepiness scale; $\mathrm{Cl}$, confidence interval. 


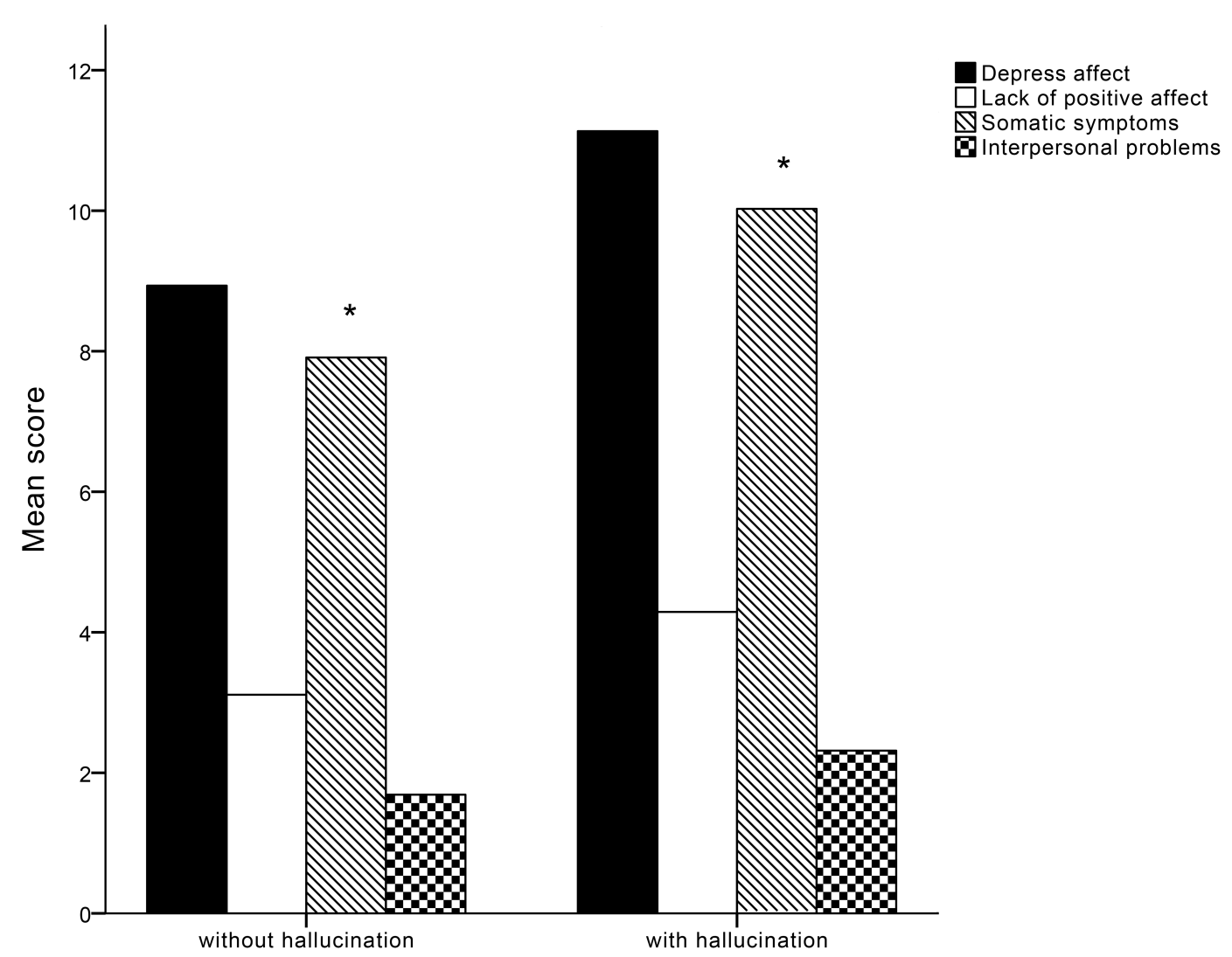

Figure 2 The mean score for sub-dimension in CES-D depression symptoms (depressed affect, lack of positive affect, somatic symptoms and interpersonal problems) between patients with hallucination and patients without hallucination. Only somatic symptoms show significant group differences $(*)$.

evaluate the potential relationship between sleep-related hallucination and depression symptoms in adolescent narcolepsy. However, there were no differences in PSG parameters between patients with and without depression symptoms in our results. It is speculated that the depression symptoms may be very mild and did not impact sleep architecture. ${ }^{17}$

Several factors may explain the higher depression symptoms reported in narcolepsy. First, hypocretin deficiency can result in mood and mental impairment. ${ }^{24}$ Hypocretin neurons widely project throughout the whole brain, including frontal lobe, limbic system, ${ }^{25}$ and orexin receptors were involved in the modulation of mood. ${ }^{26}$ Moreover, hypocretin deficiency could lead to a similar condition of cholinergic-monoaminergic imbalance in depression. ${ }^{25}$ Second, the negative effect of narcolepsy itself on life and social activity may induce depression disorder or depression symptoms in patients. ${ }^{27}$

There is rare research reporting factors associated with sub-dimensional depression symptoms. Our results showed that patients with sleep-related hallucination exhibit higher scores in depressed affect, lack of positive affect and somatic symptoms. Subjective sleepiness (ESS) was also associated with depression affect and somatic symptoms. Both subjective sleepiness and sleep-related hallucination are associated with depressed affect and somatic symptoms in narcolepsy patients. Further, patients with more severe subjective sleepiness and longer disease duration may report interpersonal problems, which may be due to the negative effect and stigma of narcolepsy. ${ }^{27}$ This should be paid more attention, especially for adolescent narcolepsy patients. Interpersonal problems can make the subject feel more isolated, having adverse effects on personality development, and more seriously, exacerbate depression feelings, resulting in suicidal thoughts. ${ }^{28}$ Although sleep parameters were not related to total depression symptoms, sleep latency and sleep efficiency were associated with depressed and positive affect, respectively. Our study shows that sleep latency comes from MSLT at the time of narcolepsy diagnosis, which represents the objective sleepiness severity in narcolepsy. ${ }^{29}$ Shorter sleep latency combined with higher ESS, which suggests more severe sleepiness, is associated with depressed affect. Reduced sleep efficiency is commonly detected in 
narcolepsy due to nocturnal sleep fragmentation. ${ }^{30}$ The current study indicated that decreased sleep efficiency is associated with positive affect deficiency, and research suggests that the positive affect is an important treatment target in depression. ${ }^{31}$ Future interventions aimed at improving sleep efficiency may be helpful in narcolepsy depression symptoms. However, with respect to the impact of depression symptoms on disease onset-age, it may be more logical to say that patients with depression symptoms have later disease onset-age, just as results in Figure 1. Patients' recalling bias and effect of depression symptoms on cognition or brain development ${ }^{32}$ should be considered when interpreting this lag in onset-age.

Limitations of this study should be mentioned. First, the cross-sectional design of this study was difficult to illustrate the long-standing relationship between narcolepsy and depression symptoms. Second, we also note that depression symptoms were assessed by patient-report measures rather than through the gold standard of clinical interviews, especially for adolescents, thus introducing the potential for under- or over-estimation of depression symptoms. Third, other factors related to depression symptoms in narcolepsy, such as insomnia, fatigue and cataplexy severity, and other comorbidities, such as attention-deficit/hyperactivity disorder and restless leg syndrome, should be considered and contributed with analyses. Finally, we did not collect data about depression symptoms from a control population and an epidemiologic survey of narcolepsy patients. Bias should be considered in more severely affected patients.

\section{Conclusion}

Sleep-related hallucination is associated with total depression symptoms in adolescent narcolepsy. Patients with depression symptoms have later disease onset-age than those without depression symptoms. For sub-dimensional depression symptoms, both sleep-related hallucination and subjective sleepiness are associated with depressed affect and somatic symptoms. Severe subjective sleepiness and longer disease duration may relate to interpersonal problems. Lower sleep efficiency is associated with a lack of positive affect and this may be a potential intervention for adolescent narcolepsy depression symptoms in the future.

\section{Acknowledgments}

We thank all the patients for their collaboration. We also thank Dr Wang Wan'er from Peking University International Hospital for the instruction in the PSG analysis.

\section{Funding}

This work was supported by the National Natural Science Foundation of China (81700088), National Natural Science Foundation of China (82070091), International Cooperation and Exchange of the National Natural Science Foundation of China (82020108001), Dongcheng District Talents Project of Beijing (DCQYYRC-789-01DR), Youth Foundation of Beijing Tiantan Hospital (No.2018-YQN-18), Youth Talent Support Project from China Association for Science and Technology, and Project 2020BD028 supported by PKU-Baidu Fund.

\section{Disclosure}

The authors declare that they have no competing interests.

\section{References}

1. Vandeputte M, de Weerd A. Sleep disorders and depressive feelings: a global survey with the Beck depression scale. Sleep Med. 2003;4 (4):343-345. doi:10.1016/S1389-9457(03)00059-5

2. Vignatelli L, Plazzi G, Peschechera F, Delaj L, D’Alessandro R. A 5-year prospective cohort study on health-related quality of life in patients with narcolepsy. Sleep Med. 2011;12(1):19-23. doi:10.1016/j.sleep.2010.07.008

3. Daniels E, King MA, Smith IE, Shneerson JM. Health-related quality of life in narcolepsy. J Sleep Res. 2001;10(1):75-81. doi:10.1046/ j.1365-2869.2001.00234.x

4. Ohayon MM. Narcolepsy is complicated by high medical and psychiatric comorbidities: a comparison with the general population. Sleep Med. 2013;14(6):488-492. doi:10.1016/j.sleep.2013.03.002

5. Ruoff CM, Reaven NL, Funk SE, et al. High rates of psychiatric comorbidity in narcolepsy: findings from the burden of narcolepsy disease (BOND) study of 9312 patients in the United States. J Clin Psychiatry. 2017;78(02):171-176. doi:10.4088/JCP.15m10262

6. Walacik-Ufnal E, Piotrowska AJ, Wołyńczyk-Gmaj D, et al. Narcolepsy type 1 and hypersomnia associated with a psychiatric disorder show different slow wave activity dynamics. Acta Neurobiol Exp. 2017;77(2):147-156. doi:10.21307/ane-2017-047

7. Zamarian L, Högl B, Delazer M, et al. Subjective deficits of attention, cognition and depression in patients with narcolepsy. Sleep Med. 2015;16(1):45-51. doi:10.1016/j.sleep.2014.07.025

8. Roy A. Psychiatric aspects of narcolepsy. Br J Psychiatry. 1976;128 (6):562-565. doi:10.1192/bjp.128.6.562

9. Lee M-J, Lee S-Y, Yuan -S-S, et al. Comorbidity of narcolepsy and depressive disorders: a nationwide population-based study in Taiwan. Sleep Med. 2017;39:95-100. doi:10.1016/j.sleep.2017.07.022

10. Dauvilliers Y, Arnulf I, Mignot E. Narcolepsy with cataplexy. Lancet. 2007;369(9560):499-511. doi:10.1016/S0140-6736(07)60237-2

11. Barateau L, Lopez R, Chenini S, et al. Depression and suicidal thoughts in untreated and treated narcolepsy. Neurology. 2020;95 (20):e2755-e68. doi:10.1212/WNL.0000000000010737

12. Glied S, Pine DS. Consequences and correlates of adolescent depression. Arch Pediatr Adolesc Med. 2002;156(10):1009-1014. doi:10.1001/archpedi.156.10.1009

13. Goodman E, Whitaker RC. A prospective study of the role of depression in the development and persistence of adolescent obesity. Pediatrics. 2002;110(3):497-504. doi:10.1542/peds.110.3.497

14. Korczak DJ, Lipman E, Morrison K, Szatmari P. Are children and adolescents with psychiatric illness at risk for increased future body weight? A systematic review. Dev Med Child Neurol. 2013;55 (11):980-987. doi:10.1111/dmcn.12168 
15. Grøholt B, Ekeberg O, Wichstrøm L, Haldorsen T. Suicidal and nonsuicidal adolescents: different factors contribute to self-esteem. Suicide Life Threat Behav. 2005;35(5):525-535. doi:10.1521/ suli.2005.35.5.525

16. Shaffer D, Gould MS, Fisher P, et al. Psychiatric diagnosis in child and adolescent suicide. Arch Gen Psychiatry. 1996;53:339-348. doi:10.1001/archpsyc.1996.01830040075012

17. Inocente $\mathrm{CO}$, Gustin MP, Lavault $\mathrm{S}$, et al. Depressive feelings in children with narcolepsy. Sleep Med. 2014;15(3):309-314. doi:10.1016/j.sleep.2013.08.798

18. Medicine AAoS. The International Classification of Sleep Disorders. 3rd ed. Darien, IL: American Academy of Sleep Medicine; 2014.

19. Berry RB, Harding SM, Brooks R; for the American Academy of Sleep Medicine. The AASM Manual for the Scoring of Sleep and Associated Events: Rules, Terminology and Technical Specifications. Darien, IL: American Academy of Sleep Medicine; 2018.

20. Zhao Z, Ding N, Song S, Liu Y, Wen D. Association between depression and overweight in Chinese adolescents: a cross-sectional study. BMJ Open. 2019;9(2):e024177. doi:10.1136/bmjopen-2018-024177

21. Vilagut G, Forero CG, Barbaglia G, Alonso J, van der Feltz-cornelis C. Screening for depression in the general population with the center for epidemiologic studies depression (CES-D): a systematic review with meta-analysis. PLoS One. 2016;11(5):e0155431. doi:10.1371/ journal.pone.0155431

22. Dauvilliers Y, Paquereau J, Bastuji H, Drouot X, Weil JS, Viot-Blanc V. Psychological health in central hypersomnias: the French Harmony Study. J Neurol Neurosurg Psychiatry. 2009;80(6):636-641. doi:10.1136/jnnp.2008.161588

23. Szklo-Coxe M, Young T, Finn L, Mignot E. Depression: relationships to sleep paralysis and other sleep disturbances in a community sample. J Sleep Res. 2007;16(3):297-312. doi:10.1111/j.13652869.2007.00600.x
24. Brundin L, Björkqvist M, Petersén A, Träskman-Bendz L. Reduced orexin levels in the cerebrospinal fluid of suicidal patients with major depressive disorder. Eur Neuropsychopharmacol. 2007;17 (9):573-579. doi:10.1016/j.euroneuro.2007.01.005

25. Sakurai T. The neural circuit of orexin (hypocretin): maintaining sleep and wakefulness. Nat Rev Neurosci. 2007;8(3):171-181. doi:10.1038/nrn2092

26. Scott MM, Marcus JN, Pettersen A, et al. Hcrtr1 and 2 signaling differentially regulates depression-like behaviors. Behav Brain Res. 2011;222(2):289-294. doi:10.1016/j.bbr.2011.02.044

27. Li X, Sanford LD, Zong Q, et al. Prevalence of depression or depressive symptoms in patients with narcolepsy: a systematic review and meta-analysis. Neuropsychol Rev. 2020;31(1):89-102. doi:10.1007/s11065-020-09443-7

28. Renner F, Jarrett RB, Vittengl JR, Barrett MS, Clark LA, Thase ME. Interpersonal problems as predictors of therapeutic alliance and symptom improvement in cognitive therapy for depression. $J$ Affect Disord. 2012;138(3):458-467. doi:10.1016/j.jad.2011.12.044

29. Aydinoz S, Huang YS, Gozal D, Inocente CO, Franco P, KheirandishGozal L. Allergies and disease severity in childhood narcolepsy: preliminary findings. Sleep. 2015;38(12):1981-1984. doi:10.5665/ sleep. 5254

30. Leger D, Tonetti L, Gauriau C, et al. A study on the optimal length of actigraphic recording in narcolepsy type 1. Clin Neurophysiol Pract. 2019;4:114-118. doi:10.1016/j.cnp.2019.04.004

31. Taylor CT, Lyubomirsky S, Stein MB. Upregulating the positive affect system in anxiety and depression: outcomes of a positive activity intervention. Depress Anxiety. 2017;34(3):267-280. doi:10.1002/da.22593

32. Disner SG, Beevers CG, Haigh EA, Beck AT. Neural mechanisms of the cognitive model of depression. Nat Rev Neurosci. 2011;12 (8):467-477. doi:10.1038/nrn3027

\section{Publish your work in this journal}

Nature and Science of Sleep is an international, peer-reviewed, open access journal covering all aspects of sleep science and sleep medicine, including the neurophysiology and functions of sleep, the genetics of sleep, sleep and society, biological rhythms, dreaming, sleep disorders and therapy, and strategies to optimize healthy sleep.
The manuscript management system is completely online and includes a very quick and fair peer-review system, which is all easy to use. Visit http://www.dovepress.com/testimonials.php to read real quotes from published authors. 